\title{
Planarer 6-Achs-Kraftsensor
}

Paul Szász, ABB Forschungszentrum DECRC/A3, Wallstadter Str. 59, 69526 Ladenburg, Deutschland, Tel. 0049-6203-716311, E-Mail: paul.szasz@de.abb.com Markus Roos, NM Numerical Modelling GmbH, Böhnirainstrasse 12, 8800 Thalwil, Schweiz, Tel. 0041-44720-1301, markus.roos@nmtec.ch Ulf Ahrend, ABB Forschungszentrum DECRC/A3, Wallstadter Str. 59, 69526 Ladenburg, Deutschland, Tel. 0049-6203-716167, E-Mail: ulf.ahrend@de.abb.com

\section{$1 \quad$ Einleitung}

6-achsige Kraftsensoren erfassen in einem Sensorkörper sowohl Kräfte in drei Raumrichtungen als auch Drehmomente um drei Raumachsen. Kommerziell verfügbare Sensoren dieser Art sind hochpräzise, komplexe und entsprechend teure Geräte. Sie bestehen typischerweise aus einem komplexen 3-dimensionalen Federkörper, der aufwändig zerspanend hergestellt werden muß (Abbildung 1). Anschließend werden auf die ausgewiesenen Federelemente Dehnmeßstreifen von Hand montiert, was wegen der erforderlichen Positionsgenauigkeit und eingeschränkter Zugänglichkeit eine langwierige und somit sehr teure Arbeit ist.
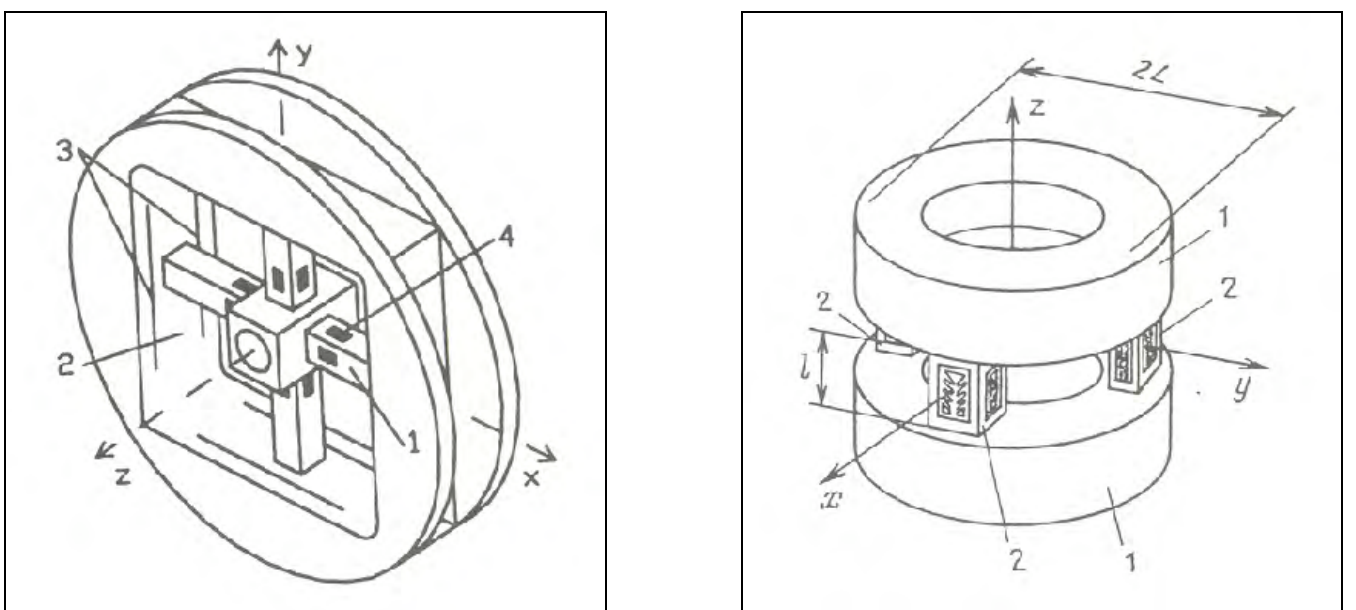

Abbildung 1: Beispiele für komplexe Strukturen von konventionellen 6-achs Kraftsensoren [1]

Die hohen Kosten beschränken die Anwendung solcher Sensoren auf wenige hochspezialisierte Bereiche, überwiegend in der Forschung und Entwicklung (z. B. Windkanalmessungen in der Flugzeugentwicklung, Lenk- und Radkraftmessungen in der Fahrzeugentwicklung u. ä.). Die in diesen Anwendungen erforderliche hohe Präzision wird durch eine aufwändige Fertigung mit hohem Handarbeitsanteil erreicht. Dies ist bei den geringen Stückzahlen und den von den Anwendern bezahlten hohen Preisen vertretbar.

Anwendungen, in denen diese hohen Preise nicht gerechtfertigt werden können, oder auch Anwendungen die große Stückzahlen benötigen würden, können von diesen Produkten nicht bedient werden. Dabei könnten z. B. in der Medizintechnik kostengünstige 6-achs-Kraftsensoren wesentliche Innovationen ermöglichen (z. B. im Bereich Rehabilitation oder Prothetik [2, 3, 4, 5]). Auch auf dem Gebiet der mechanischer Bearbeitung und Montage können solche Sensoren die Automatisierung unterstützen und zu einer hohen Reproduzierbarkeit und Qualität beitragen [6, 7]. Mit den aktuellen kommerziellen Sensoren bleiben solche Entwicklungschancen aus Kostengründen stark eingeschränkt oder ganz auf der Strecke.

\section{Idee}

Es stellt sich die Frage, ob es technisch möglich ist, einen Kraftsensor zu entwickeln, der in einer automatisierten Fertigung in großen Stückzahlen zu niedrigen Kosten gefertigt werden kann. Ein solcher Sensor würde viele Anwendungen ermöglichen, die auch mit einer mäßigen Genauigkeit des Sensors auskommen, aber nur bei niedrigen Sensorkosten rentabel sind. Dies würde eine hohe Stückzahl und damit weitere Kostensenkung ermöglichen, was zu einer Ausweitung von Kraftsensor-gesteuerten Anwendungen z. B. in der Automatisierung, Montage, Fertigung oder Medizintechnik führen kann. 
Angesichts der bekannten Aufbauten und Nachteile von üblichen 6-Achs-Kraftsensoren wurde die Idee aus zwei Forderungen abgeleitet, um die größten Schwächen der üblichen Sensoren zu beseitigen:

1. Der Federkörper muß kostengünstig herstellbar sein.

2. Der Federkörper muß automatisiert mit Dehnmeßstreifen (DMS) bestückt werden können.

\subsection{Kostengünstiger Federkörper}

Die hohen Kosten der konventionellen Federkörper werden nicht nur durch den Bedarf an speziellen Legierungen, sondern sehr stark durch die komplexe, 3-dimesionale Form und den dafür notwendigen hohen Herstellkosten geprägt. Zusätzlich zur aufwändigen zerspanenden Bearbeitung zählen hier die erforderlichen hohen Toleranzen und Oberflächengüten zu den Kostentreibern.

Eine kostengünstige Fertigung ist nur möglich, wenn die endgültige Form aus einfachem Halbzeug in einem automatisierten Verfahren in einem einfachen, schnellen Arbeitsgang herzustellen ist.

Diese Forderung wird erfüllt, wenn der Federkörper aus einem Blech konstanter Dicke mittels Laserschneiden oder Wasserstrahlschneiden hergestellt wird. Die erreichbaren Maßtoleranzen bei diesen Verfahren sind ausreichend gut, und die erforderliche Oberflächenqualität für die Dehnmeßstreifen kann bereits am Halbzeug (Blech) bereitgestellt werden, ohne aufwändige Nachbearbeitung.

\subsection{Automatisierbare Bestückung mit Dehnmeßstreifen (DMS)}

Der so erzeugte Federkörper stellt eine ebene Fläche zur Verfügung, auf der Dehnmeßstreifen in einem automatisierten Prozeß angebracht werden können. Alle Positionen sind gleich gut zugänglich. Es können moderne Bestückungstechniken verwendet werden. Auch die Direktapplikation von metallischen Dünnschicht-DMS oder keramischen Dickschicht-Widerständen ist möglich. Die Verdrahtung und sogar Teile der Elektronik können im gleichen oder direkt nachfolgenden Arbeitsgang ebenfalls automatisiert ausgeführt werden. Die Positionsgenauigkeit der einzelnen DMS kann in automatisierten Verfahren mit (gegenüber der konventionellen Handapplikation) geringem Aufwand erhöht werden.

\subsection{Geometriedefinition}

Soweit entspricht das Konzept der Herstellung von vielen einfachen, kostengünstigen einachsigen Kraftoder Drucksensoren. Entscheidend für die Funktion ist jedoch, daß die Geometrie eines solchen planaren Sensors so definiert ist, daß aus den Dehnungsmessungen aus nur einer Ebene der Struktur die volle Information über die räumliche Kraft- und Momentenverteilung gewonnen werden kann.

Auf ersten Blick erscheint es unwahrscheinlich, daß dies mit ausreichender Genauigkeit möglich ist. Es gibt zwar bereits Arbeiten die darauf hinweisen, daß bei hinreichend vielen Meßstellen aus einer Fläche (Zylinderoberfläche) die komplette 3-dimensionale Information gewonnen werden kann. Die in [8] vorgestellte Methode ist jedoch wegen der vielen Meßstellen (mehrere Dutzend) sehr aufwändig, und die zylindrische Oberfläche ist schwierig automatisch präzise zu bestücken; sie kann also die hier gestellten Anforderungen nicht erfüllen.

Deshalb wurde eine detaillierte Studie bei der NM Numerical Modelling GmbH (Thalwil, Schweiz) durchgeführt zur theoretischen Untersuchung der Fragestellung, inwieweit eine planare Struktur definiert werden kann, auf der mit einer geringen Anzahl von Meßstellen die komplette 3-dimensionale Information über die Belastung zu bestimmen ist.

\subsubsection{Grundlagen der Betrachtung}

Unter der Voraussetzung, dass beim Meßvorgang nur kleine Deformationen auftreten und das Federkörpermaterial lediglich innerhalb des linear-elastischen Bereichs belastet wird, hängt jede denkbare Messgrösse, z.B. eine Verschiebung $u^{\prime}(x)$ oder der Verzerrungstensor $\varepsilon_{i j}\left(\begin{array}{l}1 \\ x\end{array}\right)$ an der Stelle $x^{\prime}$ linear von aufgebrachten Kräften, resp. Drehmomenten ab. Dies ist eine direkte Folge der Linearität der mechanischen Bewegungsgleichungen. Betrachtet man eine Position mit Dehnmeßstreifen, der die Dehnung $\varepsilon=\sum_{i j} n_{i} \varepsilon_{i j} n_{j}$ in Richtung des Einheitsvektors $h$ misst, kann die behauptete lineare Abhängigkeit von externen Kräften folgendermassen ausgedrückt werden 


$$
\varepsilon=\sum_{k} \alpha_{k} F_{k}+\sum_{l} \beta_{l} M_{l}, k, l=1, \ldots, 3
$$

wo $F_{k}, M_{l}$ die extern aufgebrachten Kraft- resp. Drehmomentkomponenten bezeichnen. Die konstanten, reellen Koeffizienten $\alpha_{k}, \beta_{l}$ können dabei auch den Wert Null annehmen. Gleichung (1) entspricht dem Superpositionsprinzip: die kombinierte Belastung diverser Kräfte oder Momente führt zu einer additiven Überlagerung der Einzeleffekte.

Im Allgemeinen wird die Dehnung an einem Meßort durch verschiedene Kraft- und Momentkomponenten beeinflusst, d.h. meist sind die Koeffizienten in Gleichung (1) ungleich Null. In symmetrischen Objekten können ausgewählte Koeffizienten jedoch verschwinden (siehe unten). Das bedeutet, dass im Allgemeinen aus dem Messwert $\varepsilon$ nicht eindeutig auf eine einwirkende Kraft- oder Drehmomentkomponente geschlossen werden kann. Zur Messung der je 3 Kraft- und Momentkomponenten sind im Allgemeinen mindestens 6 Messwerte notwendig. Da für jede Messgrösse ein Zusammenhang gemäss Gleichung (1) gilt, kann man den Sensor mathematisch folgendermassen charakterisieren

$$
\varepsilon_{m}=\sum_{n} S_{m n} f_{n}, m=1, \ldots, n_{m}, n=1, \ldots, 6
$$

wobei $n_{m} \geq 6$ eine zu bestimmende Anzahl von Messgrössen ist. $f_{n}$ ist eine verallgemeinerte

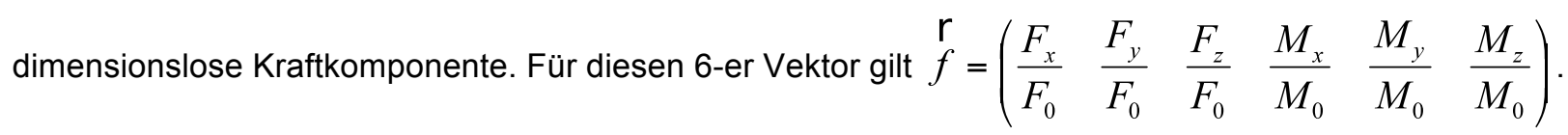

Die Skalierungswerte $F_{0}$ und $M_{0}$ für Kraft und Drehmoment werden dem gewünschten Sensormeßbereich entsprechend gewählt. So ist garantiert, dass typische Messwerte sowohl für Kraft als auch Drehmoment durch reelle Zahlen derselben Grössenordnung ausgedrückt werden. Die Elemente der Koeffizientenmatrix $S$ sind damit dimensionslos.

Die Matrix $S$ kann entweder durch Messung oder durch FE-Simulation gewonnen werden. In beiden Fällen wird vom Superpositionsprinzip Gebrauch gemacht: für die Einheitslastfälle (d.h. eine der verallgemeinerten Kraftkomponenten gleich 1, die übrigen Komponenten gleich Null) werden die Größen $\varepsilon_{m}$ gemessen, resp. simuliert. Diese Werte entsprechen der entsprechenden Spalte der Matrix $S$. Man kann sich davon überzeugen, wenn in GI (2) z.B. der Lastfall $f^{\prime}=\left(\begin{array}{llllll}1 & 0 & 0 & 0 & 0 & 0\end{array}\right)$ eingesetzt wird. Es gilt dann $\varepsilon_{m}=\sum_{n} S_{m n} f_{n}=S_{m 1} f_{1} \equiv S_{m 1}$, d.h. die $\mathrm{n}_{m}$ Dehnungen $\varepsilon_{m}$ entsprechen den S-Elementen der 1 . Spalte (analog für die übrigen Lastfälle).

\subsubsection{Mathematische Beschreibung der Meßaufgabe}

Die Meßaufgabe (Bestimmung von Kräften und Momenten aus Dehnungsmessungen) ist gelöst, wenn es gelingt, das lineare Gleichungssystem (2) nach den gesuchten verallgemeinerten Kraftkomponenten $f_{n}$ aufzulösen. Falls die Matrix $S$ quadratisch ( $n_{m}=6$ ) und regulär ist, entspricht diese Lösung formal

$$
f_{n}=\sum_{m}\left(S^{-1}\right)_{n m} \varepsilon_{m}, \text { wobei } S^{-1} \text { die Inverse der Matrix } S \text { ist. }
$$

In Worten: eine Linearkombination aller Messwerte $\varepsilon_{m}$ liefert die zu bestimmenden Kraft- und Drehmomentkomponenten, wobei die Koeffizienten der Linearkombination den Matrixelementen der Inversen der Matrix $S$ entsprechen. Ein Meßsystem mit mehr als der minimalen Anzahl von Primärmessgrössen kann analog behandelt werden. Allerdings sind dann die Komponenten der 6 Achsen überbestimmt, weshalb das Gleichungssystem nach der Methode der kleinsten Quadrate gelöst werden muss. Dieser Fall wird hier jedoch nicht weiter untersucht, d.h. wir setzen im Folgenden $n_{m}=6$ voraus, da das Ziel der Entwicklung minimale Kosten, also eine minimale Anzahl von Meßstellen ist.

Es stellen sich zwei Fragen: 
1. Unter welchen Bedingungen existiert die Inverse der Matrix $S$ ?

2. Wie stellt man sicher, dass alle 6 Achsen (Kraft und Moment) mit einer vergleichbaren Auflösung gemessen werden können?

Die Antwort auf die erste Frage ist formal einfach: die Inverse existiert, wenn die Matrix $S$ eine nicht verschwindende Determinante aufweist. Numerisch ist das jedoch meist nicht trivial. Meß- oder Simulationsfehler führen dazu, dass die Matrix $S$ nur „fast“ singulär ist. Es zeigt sich, dass man eine befriedigende Antwort auf Frage a) findet, wenn man sich zunächst Punkt b) zuwendet. Ist nämlich die Matrix $S$ fast singulär, können gewisse Komponenten nur ungenau bestimmt werden: minimale Fehler in den Primärmesswerten führen zu grossen Fehlern in den daraus bestimmten verallgemeinerten Kraftkomponenten. Aus praktischer Sicht heisst das, der Sensor weist für verschiedene Komponenten sehr stark unterschiedliche Auflösungen auf!

Um diesen Aspekt zu untersuchen, muss die Auflösung eines konkreten Systems bestimmt werden. Das gelingt mit der so genannten Singulärwertzerlegung (SVD nach dem englischen Begriff Singular Value Decomposition) der Matrix $S$. Die SVD ist eine Verallgemeinerung der Eigenwertzerlegung auf nichtsymmetrische Matrizen. Auf die Matrix $S$ angewendet, liefert die SVD

(4) $S=U \cdot D \cdot V^{T}\left(V^{T}\right.$ bezeichnet die Transponierte einer Matrix $\left.V\right)$.

$U$ und $V$ sind orthogonale $6 \times 6$-Matrizen und $D$ ist eine positiv semidefinite $6 \times 6$-Diagonalmatrix (d.h. $D_{n n} \geq 0$ ), wobei die 6 Diagnonalelemente Singulärwerte der Matrix $S$ heissen. Diese sind bis auf die Reihenfolge eindeutig. Die Faktoren der Zerlegung haben anschauliche Bedeutungen:

(4a) $\varepsilon_{n}^{\prime}=\sum_{m} U_{n m}^{T} \varepsilon_{m}$

stellt eine Linearkombination der Primärmeßwerte $\varepsilon_{m}$ dar. Die Orthogonalität der Matrix $U$ stellt sicher, dass $\varepsilon_{n}^{\prime}$ dieselbe Vektornorm aufweist, wie der entsprechende Vektor $\varepsilon_{m}$, denn $U$ ist eine verallgemeinerte Rotation im 6-dimensionalen Raum.

(4b) $f_{m}^{\prime}=\sum_{n} V_{m n}^{T} f_{n}$

ist eine Linearkombination der Lastfälle, wiederum mit unveränderter Vektornorm, weil $V$ orthogonal ist.

(4c) $\varepsilon_{m}^{\prime}=D_{m m} f_{m}^{\prime}$

verknüpft die rotierten Messwerte $\varepsilon_{n}^{\prime}$ mit den rotierten Lastfällen $f_{n}^{\prime}$. An den Diagonalelementen $D_{n n}$ kann die Sensitivität für jede rotierte Primärmeßwertkomponente abgelesen werden.

Die Diagonalwerte seien in absteigender Grösse geordnet, d.h. $D_{11} \geq D_{22} \geq \mathrm{L} \geq D_{66}$ (dies kann immer erreicht werden). Aus dem Verhältnis des grösstem zum kleinsten Wert kann auf die Meßauflösung des Systems geschlossen werden. Angenommen wir hätten $\frac{D_{66}}{D_{11}}>>1$ : die Messung von $f_{6}^{\prime}$ führt dann im Vergleich zur Messung von $f_{1}^{\prime}$ zu sehr kleinen Meßwerten $\varepsilon_{6}^{\prime}$. Damit sind auch geringe Primärmeßwerte $\varepsilon_{m}$ verbunden, wie man durch Auflösen von (4a) nach den Primärmessgrössen $\varepsilon_{m}=\sum_{m} U_{n m} \varepsilon_{n}^{\prime}$ sieht

(Orthogonalität von $U$ ). Da die technische Auflösung der Dehnmeßstreifen begrenzt ist, kann die Linearkombination $f_{6}^{\prime}$ nicht zuverlässig gemessen werden: geringe Meßfehler führen zu grossen Abweichungen in den daraus bestimmten Kraft- und Drehmomentkomponenten!

Im Umkehrschluss folgert man, dass ein ideales Meßsystem lauter identische Diagonalelemente oder jedenfalls Werte derselben Grössenordnung hat! Mit Hilfe der Matrix $D$ kann nun auch die Frage nach 
der Singularität der Matrix $S$ beantwortet werden: Ist das Verhältnis des grössten zum kleinsten Singulärwert sehr gross, können Fehler bei der numerischen Inversion der Matrix $S$ nicht vermieden werden. Man wird somit in Bezug auf numerische Stabilität der Meßprozedur auf dieselbe Bedingung geführt, wie durch die Forderung nach einer gleichmässigen Auflösung über alle Meßachsen hinweg!

\subsubsection{Auslegung des planaren Sensors}

Ein System, welches alle 6 Achsen messen kann, muss mindestens 6 DMS aufweisen. Andernfalls kann das Gleichungssystem (1) nicht aufgelöst werden! Mit den oben beschriebenen Methoden soll die Frage beantwortet werden, ob ein Sensordesign mit genau 6 Primärmesswerten aus einer Ebene, die alle sechs Kraft- bzw. Momentenkomponenten eindeutig beschreiben können, überhaupt realisierbar ist.

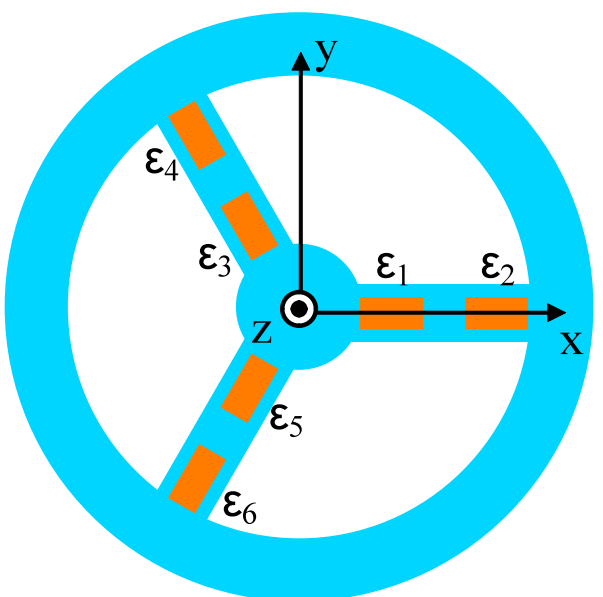

\section{Abbildung 2: Sensor mit 3-zähliger Rotationssymmetrie und 3 Spiegelachsen (übliche Anordnung)}

Zu diesem Zweck untersucht man zuerst die Struktur aus Abbildung 2, eine klassische Speichenradanordnung, wie in Mehrachs-Sensoren üblich. Allerdings werden üblicherweise alle Flächen der Speichen mit DMS bestückt, um die komplette räumliche Auflösung zu erreichen, während hier alle 6 DMS paarweise auf der Oberseite der Speichen angebracht sind (um die Forderung nach einfacher automatisierter Bestückung nachzukommen). Sowohl die Speichen als auch die DMS sind typischerweise ideal symmetrisch angeordnet. Qualitativ zeigt dieses Design folgende Charakteristik:

1. Die DMS reagieren auf Kräfte, welche in der Radebene wirken, mit Dehnung oder Kompression in den Speichen. Für eine externe Kraft in x-Richtung findet man z.B. Kompression

für $\varepsilon_{1} \approx \varepsilon_{2}<0$, und Dehnung für alle übrigen, d.h. $\varepsilon_{3}=\varepsilon_{5} \approx \varepsilon_{4}=\varepsilon_{6}>0$.

2. Eine Kraft in z-Richtung führt auf $0<\varepsilon_{1}=\varepsilon_{3}=\varepsilon_{5} \approx-\varepsilon_{2}=-\varepsilon_{4}=-\varepsilon_{6}$, da die Speichen auf Biegung beansprucht werden.

3. Drehmomente um die $x$ - und $y$-Achse in der Radebene führen ebenfalls zu Biegebeanspruchungen. Qualitativ ergibt sich für ein Moment um die x-Achse das Muster $\varepsilon_{1} \approx \varepsilon_{2} \approx 0, \varepsilon_{3} \approx \varepsilon_{4}>0$, und $\varepsilon_{5} \approx \varepsilon_{6}<0$.

4. Ein Drehmoment um die z-Achse liefert dagegen kein brauchbares Signal, da alle DMS auf Schub beansprucht werden, d.h. deren lineare Dehnung ist fast Null. In der Sprache der SVD entspricht das einer fast singulären Linearkombination!

Es stellt sich die Frage, ob dieses Verhalten durch Variation der Struktur oder alternative Anordnung der DMS korrigiert werden kann? Die Antwort lautet nein, falls Struktur und DMS dieselben Symmetrielemente aufweisen, wie in Abbildung 2, nämlich eine 3-zählige Rotationssymmetrie und 3 Spiegelebenen! Eine Symmetrietransformation der Struktur permutiert die DMS. Eine Spiegelung an der xz-Ebene in Abb. 1 liefert die Permutation der Messwerte gemäß $P_{x}=\left[\begin{array}{llllll}1 & 2 & 3 & 4 & 5 & 6 \\ 1 & 2 & 5 & 6 & 3 & 4\end{array}\right]$, also z.B. 
$\varepsilon_{1} \rightarrow \varepsilon_{1}^{\prime}=\varepsilon_{1}$, während eine $120^{\circ}$-Rotation zu $P_{r o t}=\left[\begin{array}{llllll}1 & 2 & 3 & 4 & 5 & 6 \\ 3 & 4 & 5 & 6 & 1 & 2\end{array}\right]$ führt. Liegt ein z-

Drehmoment $M_{z}>0$ an, geht dieses bei xz-Spiegelung in $M_{z} \rightarrow M_{z}^{\prime}=-M_{z}<0$ über, denn das Drehmoment ist ein Pseudovektor! Alle Messwerte $\varepsilon_{m}$ vertauschen somit ihr Vorzeichen, z.B. $\varepsilon_{1} \rightarrow \varepsilon_{1}^{\prime}=-\varepsilon_{1}$, etc. Daraus folgt $\varepsilon_{1} \equiv 0$ bei Belastung mit z-Drehmoment. Wegen der 3-zähligen Rotationssymmetrie und einer analogen Überlegung für die äusseren Dehnmeßstreifen gilt allgemein $0 \equiv \varepsilon_{1}=\varepsilon_{2}=\mathrm{L}=\varepsilon_{6}$. D.h., diese Struktur kann Drehmomente um die z-Achse nicht messen.

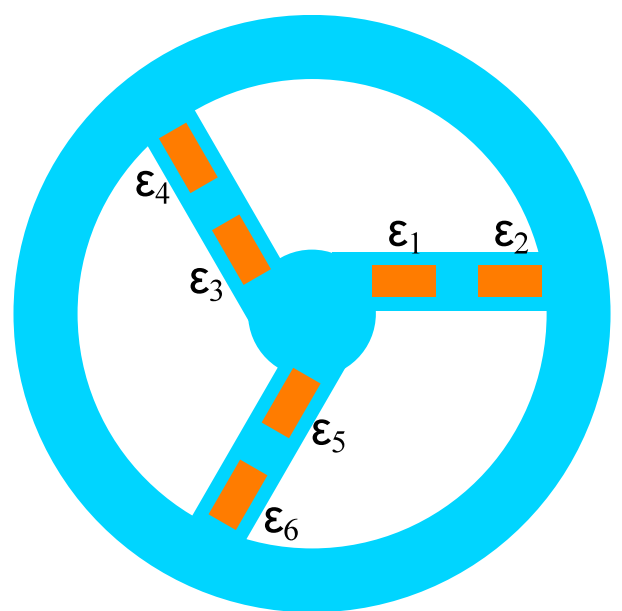

Abbildung 3: Struktur mit 3-zähliger Rotationssymmetrie ohne Spiegelachsen

Die Messung der 6 Achsen mit genau 6 Meßelementen ist dennoch möglich, wenn eine Struktur mit lediglich einer 3-zähligen Rotationssymmetrie, aber ohne Spiegelebenen gewählt wird, z. B. durch eine Verschiebung der Speichen, wie in Abbildung 3 dargestellt. Für diese Struktur ergeben sich qualitativ dieselben Abhängigkeiten für laterale und axiale Kräfte, sowie für Drehmomente um die $x$ und $y$-Achse wie bisher. Neu können damit aber auch Drehmomente um die z-Achse detektiert werden: ein Drehmoment in positiver z-Richtung führt zu Dehnungen in allen Meßelementen gemäß $0<\varepsilon_{1}=\varepsilon_{3}=\varepsilon_{5} \approx \varepsilon_{2}=\varepsilon_{4}=\varepsilon_{6}$. Man überzeugt sich leicht, dass die Meßwertmuster für alle 6 Meßachsen unterschiedlich sind. Das heißt, die zugehörige Matrix $S$ ist regulär, was durch die SVD bestätigt wird (siehe unten).

Damit wurde mindestens eine Struktur gefunden, die prinzipiell die geforderte Eigenschaft aufweist, mit nur 6 Meßwerten aus einer Ebene eindeutig alle Kraft- und Momentenkomponenten eindeutig abzubilden.

\subsubsection{Sensoroptimierung}

An der so identifizierten Struktur wurden Optimierungsrechnungen durchgeführt, um die Einflüsse der verschiedenen Geometrieparameter auf die Sensoreigenschaften zu untersuchen. Wichtige Optimierungsziele waren hierbei eine möglichst gleichmäßige Auflösung der Messung in verschiedenen Achsrichtungen, sowie eine in jeder Achsrichtung technisch ausreichend hohe Auflösung der einzelnen Dehnungen (Letzeres wird allerdings auch durch die Wahl der Meßtechnik stark beeinflußt).

Die Optimierung des Sensordesigns führt über folgende Schritte:

1. Aufbau FE-Modell.

2. Simulation der Einheitslastfälle und Bestimmung der Matrix $S$.

3. SVD von $S$, Diskussion der Diagonalwerte.

4. Wenn (zu) grosse Unterschiede vorliegen, Redesign des Sensors und zurück zu (1), sonst Ende.

Im Prinzip könnte dieser Prozedur automatisch durchlaufen werden, indem geometrische Modellparameter automatisch so modifiziert werden, dass eine Zielfunktion, welche z. B. die Unterschiede der Singulärwerte bewertet, minimal wird. Da jedoch meistens verschiedene weitere Aspekte für einen 
optimierten Sensor relevant sind, würde dies zu einer sehr komplexen Zielfunktion führen. Deshalb wird in vielen Fällen auch die Methode des „educated guess“ zu guten Resultaten führen.

Dies wurde am Beispiel der Struktur in Abbildung 3 untersucht. Um die Auflösung in allen Achsrichtungen ähnlich groß zu gestalten, muss man dafür sorgen, dass die Primärmeßwerte (Dehnungen) für die Einheitslastfälle je dieselbe Größenordnung haben. Man kann deren Niveau grob abschätzen, indem man die Speichen je nach Belastungsfall als Biegebalken oder Fachwerkstab betrachtet. Durch Variation von Hebelarmen (Durchmesser Nabe), Speichenbreite, Scheibendicke und der Position der DMS auf den Speichen kann das Verhältnis der Singulärwerte beeinflusst werden.

Natürlich ergeben sich dabei Zielkonflikte: maximale Empfindlichkeit ist nicht kompatibel mit identischer Auflösung für alle 6 Meßachsen. Für diese Optimierungsaufgabe gibt es auch kein allgemeines Optimum, nur der jeweiligen Anwendung und Anforderung angepaßte Kompromisse, die neben den Zielgrößen auch noch technische Randbedingungen (z.B. DMS-Größe, Krafteinleitungen, etc.) berücksichtigen müssen.

\begin{tabular}{|l|l|l|l|l|}
\hline \multicolumn{8}{|c|}{$D_{\text {Außen }}=50 \mathrm{~mm}, b_{\text {Speiche }}=3 \mathrm{~mm}, b_{\text {Rad }}=4 \mathrm{~mm}, \mathrm{DMS}=2 \times 4 \mathrm{~mm}$} \\
\hline$D_{\text {Nabe }}=15 \mathrm{~mm}, t=2 \mathrm{~mm}$ & \\
\hline
\end{tabular}

\section{Abbildung 4: Optimierung der Sensorstruktur}

Als Beispiel für zwei unterschiedliche Optimierungsergebnisse sind in Abbildung 4 zwei Strukturen dargestellt (beide prinzipiell entsprechend Abbildung 3), welche sich durch Nabendurchmesser $D_{\text {Nabe }}$ und Strukturdicke $t$ unterscheiden. Die Speichen sind jeweils tangential zur Nabe angeordnet und die Dehnmeßstreifen darauf extremal angeordnet. Die Singulärwerte können approximativ mit den Einheitslastfällen in Beziehung gesetzt. Wie man Abbildung 4 entnehmen kann, verbessert sich das Verhältnis von maximalem zu minimalem Singulärwert von 38 für Variante01 (links) zu 11.4 für Variante03 (rechts). Das wurde jedoch mit einer Reduktion der maximalen Empfindlichkeit von 72 auf 14 "erkauft". Es zeigt sich, dass in Variante03 das Verhältnis der Empfindlichkeit für z-Drehmomente zu zKräften grösser wurde im Vergleich zu den entsprechenden Sensitivitäten in Variation01. Wegen der Veränderung von Hebelarm (=Nabendurchmesser) und Biegebalkenlänge (=Speichenlänge) ist dieses Verhalten qualitativ nachvollziehbar.

Im Rahmen der Optimierung wurden mehrere Strukturen generiert, die ein Verhältnis der Singulärwerte von ca. 8 - 10 aufweisen bei akzeptablen Auflösungen der einzelnen DMS. Konventionelle 3-dimensional bestückte Kraftsensoren erreichen Singulärwert-Verhältnisse von ca. 3. Damit konnte gezeigt werden, daß die planaren Kraftsensoren nach dem hier gezeigten Prinzip trotz der etwas ungünstigeren Empfindlichkeitsverteilungen ausreichend empfindlich alle Achsrichtungen auflösen können, um für mäßig anspruchsvolle Anwendungen einsetzbar zu sein. 
Das oben hergeleitete Konzept des planaren Sensors wurde an einem realen Demonstrator überprüft. Ziel war es einerseits die Ergebnisse des mathematischen Modells zu überprüfen, andererseits gleichzeitig einen Technologievergleich verschiedener Dehnmeßstreifen-Lösungen durchzuführen.

\subsection{Demonstrator-Entwurf}

Als Geometrie wurde die Ausgestaltung entsprechend Abbildung 5 festgelegt. Der Demonstrator entspricht im Lastbereich wie in der Dimension etwa einem ATI Mini40, der als Vergleichsgerät vorhanden war (ca. $60 \mathrm{~N}$ und $1 \mathrm{Nm}$ Maximallast).

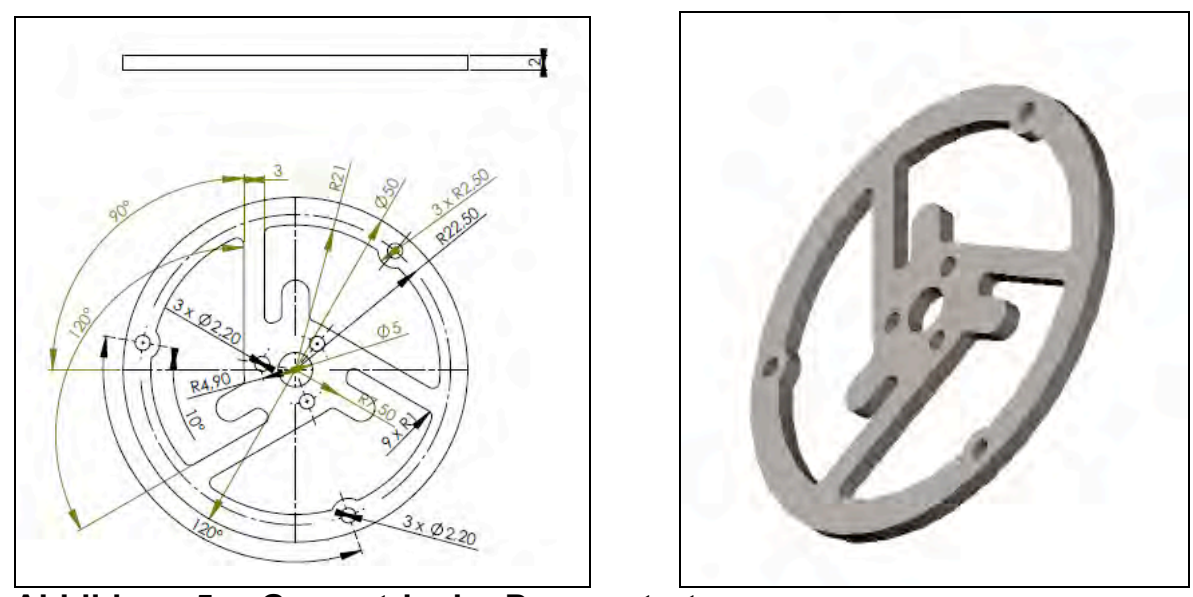

Abbildung 5: Geometrie der Demonstratoren

Ein einfaches Gehäusekonzept wurde auch entworfen, um ein Beispiel für den Einbau und die Krafteinleitung zu liefern (s. Abbildung 6). Natürlich muß so ein Gehäuse der jeweiligen Anwendung angepaßt sein, sowohl bzgl. Anschlußgeometrien als auch der Abdichtung. Zusätzlich muß das Gehäuse noch mechanische Überlastsicherung gewährleisten, was im ersten Entwurf noch nicht enthalten war.
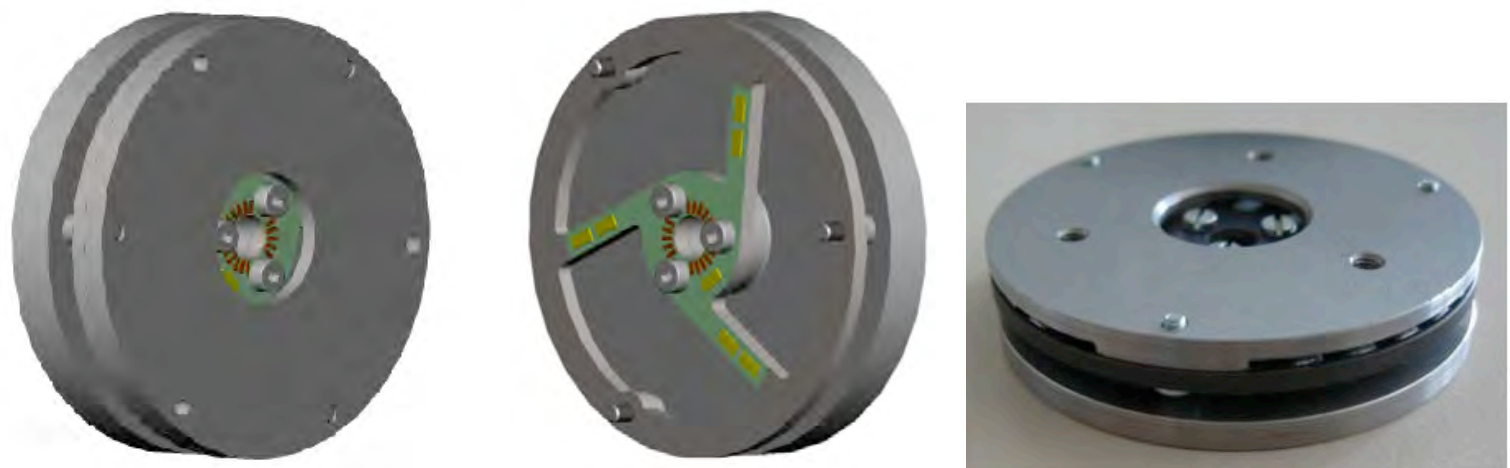

Abbildung 6: Gehäusekonzept Beispiel (links geschlossen, mitte offen, mit sichtbarem Federkörper, rechts realer Demonstrator)

\subsection{Sensor-Technik}

Zur Messung der Verformungen wurden drei unterschiedliche Dehnmeßstreifen-Technologien (DMS) untersucht, um sie bezüglich Performance, Montage und Kosten vergleichen zu können.

\subsubsection{Metallische Folien-DMS}

Diese Technik ist zu geringsten Kosten und weit verbreitet verfügbar. Den Vorteilen der geringen Materialkosten und bewährt geringer Temperaturempfindlichkeit dieser DMS stehen allerdings für einige, für unsere Anwendung wesentliche Nachteile (wie z.B. mäßige Empfindlichkeit, hoher Montageaufwand in Handarbeit) gegenüber. Die geringe Empfindlichkeit kann je nach Federkörperauslegung akzeptabel sein, und kann teilweise durch beidseitige Bestückung und Anwendung von Brückenschaltungen ausgeglichen werden. Der Handarbeitsaufwand für mindestens 6 DMS ist für ein kostengünstiges Produkt jedoch nicht akzeptabel (sowohl wegen Kosten, als auch wegen der Qualitätsrisiken z. B. bzgl. Positionsgenauigkeit). 
Um hier Abhilfe zu schaffen wurde ein Integral-DMS entworfen, auf dem die relative Position der DMS untereinander bereits in der Vorfertigung präzise garantiert wird, und das sogar die Verdrahtung der DMS beinhaltet. Dieser DMS kann kostengünstig in einem Arbeitsgang auf dem Federkörper montiert werden, was zu einem sehr gut reproduzierbaren System führt. Solche „custom-made“ DMS können bei Dimensionen bis zu einigen $\mathrm{cm}$ wirtschaftlich sein, wenn man nicht nur Materialkosten sondern auch die Montagekosten betrachtet. Für die Demonstratoren wurden solche Integral-DMS-Muster als Sonderserie hergestellt (Abbildung 7). Ein Konzept zur effektiven und präzisen Applikation wurde zusammen mit einem erfahrenen Partner ausgearbeitet und soll noch in der Praxis verifiziert werden.
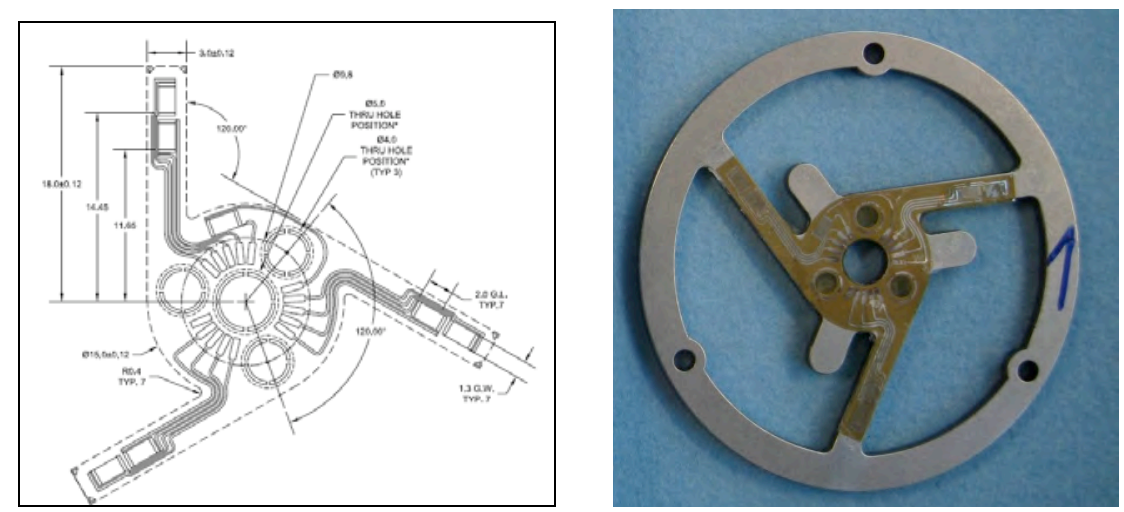

Abbildung 7: Integral-DMS für planaren Sensor - Entwurf und Demonstrator mit Integralfolie

\subsubsection{Halbleiter-DMS}

Halbleiter-DMS haben den großen Vorteil einer vielfach höheren Empfindlichkeit über Folien-DMS (bis zu Faktor 50). Das ermöglicht nicht nur eine höhere Auflösung und Genauigkeit der Messung, sondern auch alternativ eine Auslegung mit großen Lastreserven, was entweder wegen Überlastsicherheit oder wegen Verringerung von Kriech- und Hysterese-Effekten nützlich sein kann. Halbleiter-DMS können möglicherweise bei entsprechender Vorbereitung auch mit automatisierten Bestückungstechniken verarbeitet werden. Allerdings stehen diesen Chancen deutlich höhere Kosten, um Größenordnungen höhere Temperaturempfindlichkeit und eine eigeschränkte Verfügbarkeit gegenüber. Tatsächlich werden solche DMS im Sensorbau relativ selten verwendet (z. B stattet die Firma ATI ihre Kraftsensoren mit solchen DMS aus, um eine besonders hohe Überlastfähigkeit, teilweise ohne zusätzlichen mechanischen Überlastschutz, zu erreichen). Entsprechend gibt es auch nur wenige Lieferanten für solche DMS.

Für die Demonstratoren wurden relativ kostengünstige Halbleiter-DMS aus Massenfertigung eingesetzt. Da sich für die Demonstratoren eine Verfahrensentwicklung für automatisierte Bestückung nicht lohnte, wurden die Halbleiter-DMS vom DMS-Hersteller selbst in Handarbeit appliziert. Für einen besseren Vergleich wurden im gleichen Prozeß auch Demonstratoren mit konventionellen Folien-DMS hergestellt.

Die so von Hand hergestellten Demonstratoren waren gut geeignet, die Meßfähigkeit der verschiedenen Systeme zu vergleichen. Sie zeigten aber auch durch die große Streuung der Positionen und die Ungleichmäßigkeiten der Hand-Verdrahtung (Abbildung 8) die Grenzen der Handapplikaton auf und belegen damit die Notwendigkeit einer automatisierten, reproduzierbaren Montage.

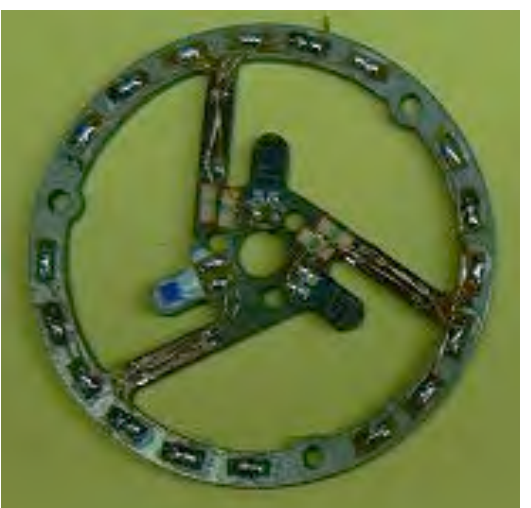

Abbildung 8: Halbleiter-DMS - Applikation und Verdrahtung in Handarbeit 


\subsubsection{Keramische Dickschicht-DMS}

Als Beispiel einer voll automatisierbaren DMS-Struktur-Applikation wurden keramische Dickschicht-DMS untersucht. Neue Entwicklungen ermöglichen es, Dickschicht-Strukturen, die üblicherweise auf KeramikSubstrate aufgetragen werden, auch auf Metallträger anzuwenden. Eine beispielhafte Anwendung wurde 2010 am Muster eines planaren mehrachsigen Kraftsensors bereits veröffentlicht [9]. Die DickschichtDMS zeichnen sich durch eine sehr gute Drift-Stabilität und eine gute Empfindlichkeit aus (über der von Folien-DMS, wenn auch geringer, als bei Halbleiter-DMS). Allerdings gibt es damit noch relativ wenig Erfahrungen, sowohl was Fertigungstechnik als auch Langzeitanwendung angeht.

Die mit diesem Verfahren hergestellten Sensoren (Abbildung 9) erwiesen sich als vielversprechend, insbesondere wegen ihrer guten Positions-Reproduzierbarkeit, guten Empfindlichkeit und der geringen Drift. Sie zeigten klar die Vorteile eines automatisierbaren Applikationsprozesses auf.
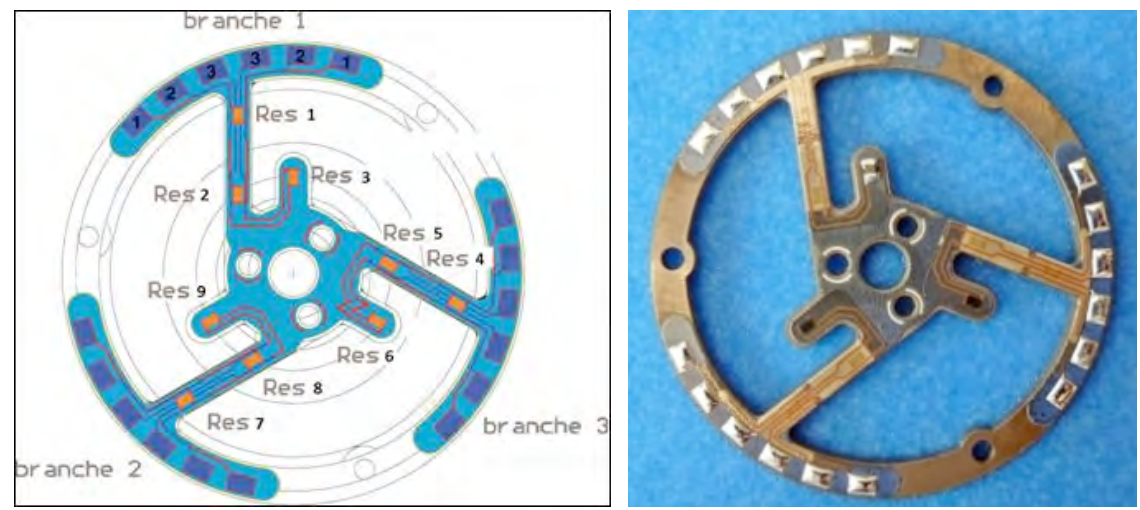

Abbildung 9: Layout und Demonstrator mit keramischen Dickschicht-DMS

\subsection{Tests und Kalibrierung}

Die Demonstratoren der verschiedenen Sensortechnologien wurden im Hinblick auf kostengünstige Montage und effektive Messung analysiert. Zusätzlich wurde der experimentelle Nachweis erbracht, daß der planare Sensor die Fähigkeit hat, die räumliche Information über alle Kräfte und Momente zu liefern.

Die Messungen erfolgten in einer einfachen Belastungsvorrichtung (Abbildung 10) unter Nutzung eines kommerziellen ATI Mini40 Sensors als Referenz. Da nur Rohdaten mit Laborelektronik ausgewertet wurden, sind die Ergebnisse natürlich nicht mit den Eigenschaften kommerzieller Sensoren mit angepaßter Elektronik (z. B. bzgl. Temperaturkompensation) vergleichbar und haben keine quantitative Aussagekraft über die tatsächliche Genauigkeit in einem realen Sensor. Die Daten erlauben aber einen guten Vergleich der Technologien untereinander und den Ausschluß von hohen Risiken.
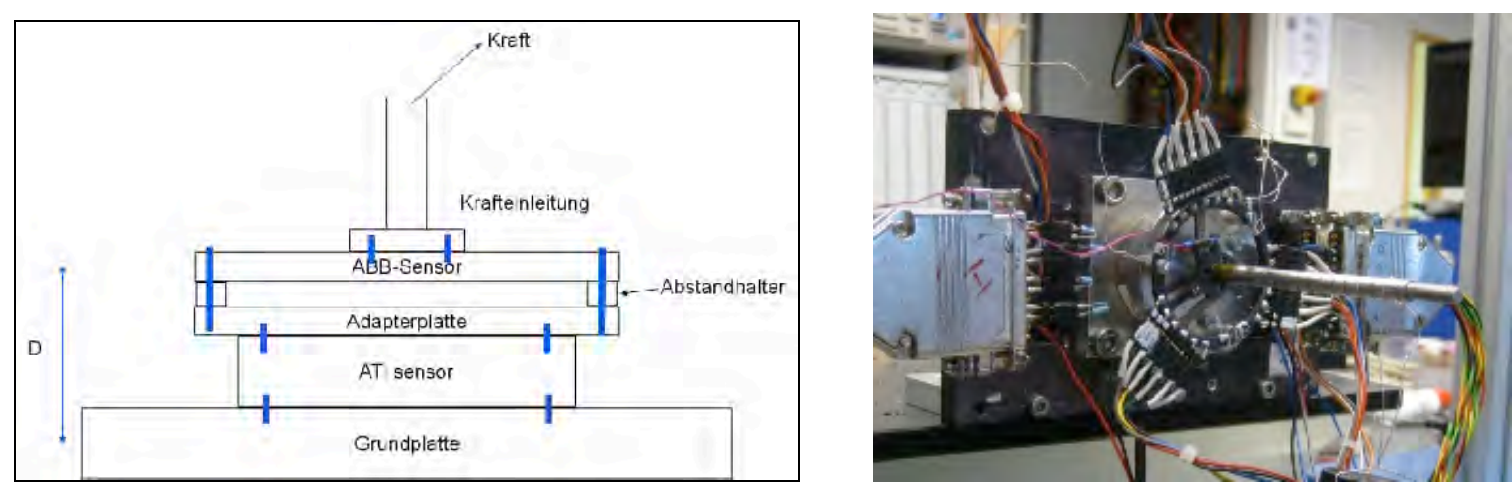

Abbildung 10: Belastungsvorrichtung für Demonstratorprüfungen

Alle überprüften DMS-Technologien erwiesen sich als prinzipiell nutzbar, wenn auch mit unterschiedlichem Potential. Folien-DMS kommen z. B. nur als Integral-Folie für einen „low-cost"-Sensor in Frage, was ihre Anwendung auf relativ geringe Sensordimensionen beschränkt. Für maximale Auflösung sind sicherlich Halbleiter-DMS erforderlich, für die jedoch der Aufwand für Kompensation der Temperaturempfindlichkeit groß ist. Die Keramik-Dickschicht-DMS zeigten ein sehr ausgewogenes Verhalten mit idealen Voraussetzungen für eine kostengünstige Serienproduktion. Wegen der eingeschränkten Erfahrung mit diesem System sind aber dafür noch weitere Untersuchungen nötig. 
Um nachzuweisen, daß die theoretische Überlegung zur Darstellung aller Belastungen aus den 6 DMSSignalen aus einer Ebene tatsächlich gilt, wurde eines der Demonstratoren für alle Achsrichtungen kalibriert. Hierfür wurde das im folgenden beschriebene Kalibrierverfahren definiert, bei dem eine Vielzahl von unterschiedlichen Belastungen in beliebiger Richtung aufgebracht wird, und die erzeugten DMSSignale zu den Vergleichssignalen eines kalibrierten Sensors korreliert werden.

Der Kalibrierprozeß basiert auf einigen Annahmen, die mit ausreichender Genauigkeit erfüllt wurden:

1. Es gibt einen linearer Zusammenhang zwischen DMS-Meßdaten und Kraft $\vec{d}=M * \vec{f}$

2. Der Sensor wird keiner plastischen Verformung unterworfen, es gibt im Allgemeinen keine Nichtlinearitäten (steifer Versuchsaufbau, geringe Verformung bzw. keine Überlast)

3. Konstante Temperatur (Durchführung in der Klimakammer)

Das Ziel des Kalibrierprozesses ist es, aus Widerstandsänderungen des zu kalibrierenden Sensors $(\vec{d})$ die Kraft- und Drehmomentwerte $(\vec{f})$ berechnen zu können, also den linearen Zusammenhang $\vec{d}=M * \vec{f}$ nach $(\vec{f})$ aufzulösen. Dies geschieht mittels „Pseudoinversen“. Da die Kopplungsmatrix $M$ (weil ihre Symmetrie nicht gewiss ist) nicht einfach invertiert werden kann, wird sie zunächst mit ihrer Transponierten $M^{T}$ multipliziert $\left(M^{T} * \vec{d}=\left(M^{T} * M\right) * \vec{f}\right)$ und anschließend invertiert: $\left.\left(M^{T} \cdot M\right)^{-1} \cdot M^{T} \cdot \stackrel{\mathrm{I}}{d}=\stackrel{\mathrm{I}}{f}\right)$. Damit ist die Auflösung nach $\vec{f}$ erfolgt. Gesucht ist folglich die Entkopplungsmatrix $R_{f}=\left(M^{T} \cdot M\right)^{-1} \cdot M^{T} \cdot \stackrel{\mathrm{I}}{d}=\stackrel{\mathrm{I}}{f}$ ) die die Gleichung $R_{f} * \vec{d}=\vec{f}$ erfüllt.

Um $M$ oder $M^{T}$ zu bestimmen werden eine Reihe von $m$ Messungen mit verschiedenen Lastfällen $\vec{f}_{m}$ aufgenommen, die jeweils $m$ Datensätze $\vec{d}_{m}$ generieren. Die gesamte Kalibrier-Meßreihe kann dann folgendermaßen zusammengefaßt werden:

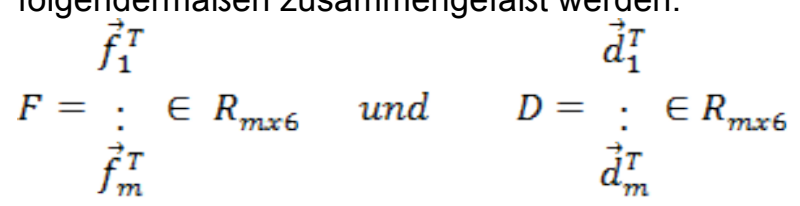

Für die einzelne Messung gilt jeweils:

Und somit: für die gesamte Meßreihe

$\vec{d}^{T}=(M * \vec{f})^{T}=\vec{f}^{T} * M^{T}$

$D=F * M^{T}$.

Mit Hilfe der Pseudoinversen erhält man:

$M^{T}=\left[\left(F^{T} * F\right)^{-1} * F^{T}\right] * D$

Es kann also anhand der Meßdaten der (mit einem kalibrierten Referenzsensor gemessenen) tatsächlichen Kräfte und Momente und den Signalen des zu kalibrierenden Sensors die Kopplungsmatrix $M$ berechnet werden und daraus anschließend auch die Entkopplungsmatrix $R_{f}$. Die Genauigkeit der Kalibrierung ist umso besser, je besser die Eingangsannahmen erfüllt sind, und je höher die Zahl $m$ der Messungen ist, wobei letzterer Parameter natürlich auch einen Kostenfaktor darstellt. Der Vorteil dieses Verfahrens ist, daß die Belastungen keine besonderen Anforderungen erfüllen müssen (z. B: bezüglich Belastungsrichtung). Somit sind auch keine besonders aufwändigen, präzisen Belastungsvorrichtungen notwendig.

In den durchgeführten Versuchen konnte gezeigt werden, daß mit einem vertretbaren Meßaufwand eine Entkopplungsmatrix $R_{f}$ berechnet werden konnte, (üblicherweise „Kalibriermatrix“ genannt), welche mit ausreichender Genauigkeit bei anschließenden Messungen die tatsächlichen Kräfte und Momente berechnen läßt. Damit konnte experimentell nachgewiesen werden, daß das planare Konzept für einen 6Achs-Kraftsensor tatsächlich umsetzbar ist. Weitere Arbeit ist noch erforderlich, um den Kalibrierprozeß zu optimieren, z. B. um die Zahl der Messungen auf die erforderliche Genauigkeit abzustimmen und die Gültigkeit der Kalibriermatrix über längere Zeit zu verifizieren (Effekte von Kriechen, Alterung, Drift, etc.). 


\section{$4 \quad$ Zusammenfassung und Ausblick}

Das Konzept der planaren mehrachsigen Kraftsensoren wurde theoretisch entwickelt und durch erfolgreiche Kalibrierung an Demonstratoren nachgewiesen. Mehrere DMS-Technologien wurden daran untersucht und ihre Eignung wie auch ihre technischen Grenzen wurden bestimmt. Bei der Herstellung der Demonstratoren wurden verschiedene Möglichkeiten von automatisierten Fertigungsverfahren für hohe Reproduzierbarkeit und niedrige Kosten verifiziert.

Damit ist das (zum Patent angemeldete) Konzept bereit zur Umsetzung in eine Technologieentwicklung für konkrete Produkte. Die zugehörige Elektronikentwicklung wird als risikoarm eingestuft. Je nach definierter Anwendung ist natürlich ein geeignetes Packaging (z. B. Gehäuse, Überlastschutz, Abdichtung) zu entwickeln. Dabei kann auf vorhandene Technologien und Lösungen aus dem Kraftsensor-Bau zurückgegriffen werden.

Eine solche Technologieentwicklung ist besonders erfolgversprechend, wenn eine passende Anwendung bzw. Produkt identifiziert wird, das einerseits keine sehr hohe Anforderungen an Genauigkeit stellt, und andererseits ausreichende Stückzahlen bietet, um die anvisierten (teil-)automatisierten Fertigungsverfahren zu rechtfertigen.

\section{$5 \quad$ Literatur}

[1] D. Grinevsky, A. Formalsky, A.Schneider: Force Control of Robotics Systems, CRC Press LLC, 1997

[2]: Robotics for Rehabilitation, Factors for Success from a European Perspective, Christian Bühler, FTB, http://www.asel.udel.edu/ robotics/newsletter/sprsum97/europe.html, Last updated: November 1997, Copyright @ Applied Science and Engineering Laboratories, 1997.

[3]: Dallaway JL, Jackson RD, Timmers PHA (1995) Rehabilitation Robotics in Europe. IEEE Transactions on Rehabilitation Engineering. 3. 35-45.

[4]: Marktstudie: Robotic Surgery - Large Potential Market, http://www.markt-studie.de/studien/roboticsurgery-large-potential-market-p-24009.html\#anker0

[5]: Proceedings of 12TH INTERNATIONAL CONFERENCE ON REHABILITATION ROBOTICS, June 29 - July 1, 2011, ETH Zurich, Switzerland

[6]: Flex Finishing - kraftsteuerung für Roboterbearbeitungsprozessehttp: http://www02.abb.com/global/atabb/atabb104.nsf/0/faf35894d2549990c125735500304772/\$file/RO BOTIC B ForceControl+f\%C3\%BCr+Bearbeitungsprozesse deu low res.pdf [7]:Intelligent Robots with FANUC Force Sensors: http://www.fanucrobotics.com/file-repository/DataSheets/Robots/Intelligent-Robots-with-FANUCForce-Sensors.pdf

[8]: Barry Spletzer and Lisa Marron, Sandia National Laboratories: Information Package for the Simplified Six-Axis Load Cell, January 2000, http://prod.sandia.gov/techlib/accesscontrol.cgi/2000/000695.pdf, Patent US5850044

[9]: C. Jacq, et al., Thick-film multi-DOF force/torque sensor for wrist rehabilitation, Sens. Actuators A: Phys. $(2010$ 